\title{
Predicting atomic dopant solvation in helium clusters: The $\mathrm{MgHe}_{n}$ case
}

\author{
Massimo Mella ${ }^{a}$ \\ School of Chemistry, Cardiff University, Main Building, Park Place, Cardiff CF10 3AT, United Kingdom \\ Gabriele Calderoni ${ }^{\mathrm{b})}$ \\ Dipartimento di Chimica Fisica ed Elettrochimica, Università degli Studi di Milano, via Golgi 19, 20133 \\ Milano, Italy \\ Fausto Cargnonic) \\ Istituto di Scienze e Tecnologie Molecolari-Consiglio Nazionale delle Ricerche (ISTM-CNR), via Golgi 19, \\ 20133 Milano, Italy
}

(Received 3 February 2005; accepted 2 June 2005; published online 10 August 2005)

\begin{abstract}
We present a quantum Monte Carlo study of the solvation and spectroscopic properties of the Mg-doped helium clusters $\mathrm{MgHe}_{n}$ with $n=2-50$. Three high-level [MP4, CCSD(T), and CCSDT] $\mathrm{MgHe}$ interaction potentials have been used to study the sensitivity of the dopant location on the shape of the pair interaction. Despite the similar $\mathrm{MgHe}$ well depth, the pair distribution functions obtained in the diffusion Monte Carlo simulations markedly differ for the three pair potentials, therefore indicating different solubility properties for $\mathrm{Mg}$ in $\mathrm{He}_{n}$. Moreover, we found interesting size effects for the behavior of the Mg impurity. As a sensitive probe of the solvation properties, the Mg excitation spectra have been simulated for various cluster sizes and compared with the available experimental results. The interaction between the excited ${ }^{1} P \mathrm{Mg}$ atom and the He moiety has been approximated using the diatomics-in-molecules method and the two excited ${ }^{1} \Pi$ and ${ }^{1} \Sigma \mathrm{MgHe}$ potentials. The shape of the simulated $\mathrm{MgHe}_{50}$ spectra shows a substantial dependency on the location of the $\mathrm{Mg}$ impurity, and hence on the $\mathrm{MgHe}$ pair interaction employed. To unravel the dependency of the solvation behavior on the shape of the computed potentials, exact density-functional theory has been adapted to the case of doped $\mathrm{He}_{n}$ and various energy distributions have been computed. The results indicate the shape of the repulsive part of the MgHe potential as an important cause of the different behaviors. (C) 2005 American Institute of Physics.
\end{abstract}

[DOI: $10.1063 / 1.1982787]$

\section{INTRODUCTION}

The cold and gentle environment represented by bulk ${ }^{4} \mathrm{He}$ and by ${ }^{4} \mathrm{He}$ clusters has been attracting the attention of the physical chemistry community due to very peculiar properties such as the absence of internal friction, the small interaction energy with a doping impurity, and the ability to dissipate promptly the excess energy of excited molecules (see Ref. 1 for an extensive review on these subjects). These features make the He nanodroplets an ideal environment to carry out reactions without the kinetic bottleneck represented by the time needed by the reactants to diffuse and collide, and to record high accuracy spectra of ultracold molecules and molecular complexes.

Despite the fact that the interaction energy between $\mathrm{He}$ atoms and the doping impurities is usually very small, such interaction plays an important role in many interesting quantum phenomena. As an example we recall the "adiabatic following" of the molecular rotations by the neighboring $\mathrm{He}$ atoms. ${ }^{2}$ This effect induces the increase of the momentum of inertia of molecules solvated by helium, and is experimen-

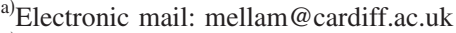

b) Electronic mail: gabriele.calderoni@unimi.it

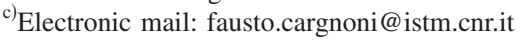

tally detected by the decrease of the spacing between the absorption lines in the microwave spectrum. ${ }^{1}$

Due to the highly quantum nature of He aggregates, even the much simple solvation process of a neutral impurity is not completely rationalized, and up to now only few attempts to reach a detailed description of the experimental findings have been carried out. ${ }^{3,4}$ Among the unsolved issues, the subtle interplay between the various parameters of the system playing a role in the solvation mechanism (i.e., the features of the interaction potential between helium and the impurity) still waits to be fully uncovered.

An attempt in this direction has been made by Ancillotto et al. ${ }^{5}$ These authors modeled superfluid helium by means of a density-functional theory (DFT) approach, using an approximate energy functional. Assuming an infinite atomic mass for the impurity, and that its interaction energy curve with helium takes the form of a Lennard-Jones potential, they reached a clear-cut description of the solvation phenomenon in terms of the single dimensionless parameter $\lambda$, defined as

$$
\lambda=2^{-1 / 6} \sigma^{-1} \rho \epsilon r_{e},
$$

where $\sigma$ is the surface tension of liquid He, $\rho$ is the number density of bulk He, and $\epsilon$ and $r_{e}$ are the well depth and the equilibrium distance of the He-impurity potential, respec- 
tively. The computed value of $\lambda$ unambiguously discriminates between opposite behaviors. Indeed, if $\lambda$ is larger than 1.9 the free energy of the impurity decreases as it moves from the surface to the bulk helium, indicating the onset of the solvation process. Conversely, if $\lambda<1.9$ the minimum free energy is reached when the impurity resides on the surface of the bulk, and thus no solvation occurs.

Despite the merits of simplicity, and of reducing the number of independent variables to a single one, this model was not devised to describe in detail the solvation process, but only to predict whether for a given impurity the solvation occurs or not. Furthermore, the DFT approach does not take into account properly the discrete nature and the anisotropic deformation $^{6,7}$ of the He aggregates, and this might lead to overestimate the overall interaction energy with the impurity. The drawbacks of this approximation, as well as the ones cited above, are expected to be particularly relevant in systems with $\lambda$ close to the critical value of 1.9 .

A deep description of the solvation process can be gained by solving exactly the Schrödinger equation of nuclear motion using an explicit many-body algorithm. In systems where accurate interaction potentials between helium and the impurity are available, the quantum Monte Carlo (QMC) approach is probably the best suited, and has been already applied successfully to the study of doped helium clusters. ${ }^{6-12}$ Though the diffusion Monte Carlo (DMC) simulations cannot recover the temporal evolution of the system, they provide many important quantities that are hardly accessible experimentally, such as radial and angular distribution functions, solvation energies, excitation spectra, as well as their dependence on the size of the helium aggregate.

In the present study we applied this method to study the doping of helium clusters with a neutral $\mathrm{Mg}$ atom, a system that in recent years has been the subject of two experimental investigations. ${ }^{13,14}$ Moriwaki and Morita $^{13}$ measured the emission and the excitation spectra of $\mathrm{Mg}$ dispersed in liquid helium by means of ultraviolet laser excitation, and focused their attention on the signals arising from the $\operatorname{Mg}\left(3 s^{2}\right)^{1} S$ $\rightarrow \operatorname{Mg}(3 s 3 p){ }^{1} P$ transition. Reho et al. ${ }^{14}$ studied this same transition of $\mathrm{Mg}$ while interacting with helium nanodroplets. Based on the comparison with the available experimental data for $\mathrm{Mg}$ (Ref. 13) and for other neutral metal atoms in helium, Reho et al. concluded that $\mathrm{Mg}$ is indeed solvated by helium, and thus does not reside on the surface of the nanodroplets. However, they stressed that no clear-cut description of this system can be recovered by theory using the model proposed by Ancillotto et al. ${ }^{5}$ because the $\lambda$ values computed from the available model potentials for $\mathrm{Mg}-\mathrm{He}$ are heavily scattered around the critical value of 1.9 (see Refs. 15 and 16, and Ref. 14 for an exhaustive review up to 1999); furthermore, the performance of this scheme in nearly critical conditions (i.e., for $\lambda$ values close to 1.9) still needed to be assessed.

To shed light on these arguments is the main scope of the present work. As a first step we generated accurate potentialenergy surfaces (PESs) for the interaction between a $\mathrm{Mg}$ atom in the ground state and $\mathrm{He}$ using the MP4 and $\operatorname{CCSD}(\mathrm{T})$ methods, and for $\mathrm{Mg}$ in the ${ }^{1} P$ state and He with a multiconfigurational approach, adopting for all computations high-quality basis sets. The PESs of the excited Mg-He complex are necessary to compute the excitation spectrum of $\mathrm{Mg}$ attached to He clusters. While our investigation was on its way, two alternative potentials for the ground-state $\mathrm{Mg}-\mathrm{He}$ interaction were proposed, based on CCSD(T) (Ref. 16) and CCSDT (Ref. 17) computations. The interaction energies computed by Partridge et al. ${ }^{16}$ were nearly superimposable to our $\operatorname{CCSD}(\mathrm{T})$ potential, while the ones by Hinde ${ }^{17}$ turned out to be noticeably different from both potentials computed by us; therefore we extended our investigation by adopting also Hinde's proposal.

Second, we detailed the solvation phenomenon by performing DMC simulations on $\mathrm{MgHe}_{n}$ clusters with growing size $(n=2-50)$; this approach helps in highlighting possible size effects that are difficult to probe experimentally. Since the manifolds of the excitation spectrum largely depend on the onset of solvation, we also computed the PES for the three low-lying $\operatorname{Mg}(3 s 3 p)-\mathrm{He}$ excited states. Their implementation in DMC simulations according to the diatomics-in-molecules $^{18}$ (DIM) scheme allowed us to recover the excitation spectrum of magnesium interacting with helium clusters, and thus to compare directly theoretical with available experimental results.

The outline of this work follows. Section II presents the details of the $a b$ initio computations performed to determine the ground- and excited-states two-body $\mathrm{Mg}-\mathrm{He}$ interaction potentials, with a short discussion on the relevance of the three-body effects in $\mathrm{MgHe}_{n}$ clusters. In Sec. III we give a short introduction to the quantum Monte Carlo methods used in this work to solve the Schrödinger equation for nuclear motion. In Sec. IV we present the results of the DMC simulations, and compare our data with the available experimental measurements. ${ }^{13,14}$ Finally, Sec. V reports our conclusions, along with a prospect on future applications.

\section{INTERACTION POTENTIALS}

The availability of accurate interaction potentials is a prerequisite for a reliable modeling of doped helium clusters. In the present investigation, the complete PESs of the ground- and excited-states $\mathrm{Mg}-\mathrm{He}_{n}$ clusters are approximated by means of two-body terms. Within this approach the interaction energy in the ground-state clusters is predicted simply by summing up all the pairwise $\operatorname{Mg}\left({ }^{1} S\right)-\mathrm{He}$ and $\mathrm{He}-\mathrm{He}$ contributions. Conversely, in the excited clusters we adopted the DIM formalism ${ }^{18}$ to recover the overall $\mathrm{Mg}-\mathrm{He}_{n}$ interaction energy from the computed two-body $\operatorname{Mg}\left({ }^{1} P\right)-\mathrm{He}$ potentials.

As concerns the He-He interaction, we selected the Tang-Toennies-Yiu (TTY) potential proposed by Tang et al., ${ }^{19}$ which is established as one of the most accurate PESs for this system.

In the case of the ground-state $\mathrm{Mg}-\mathrm{He}$ complex, a number of interaction energy curves were proposed in the last 15 years. ${ }^{14,16,17,20-23}$ The most accurate were computed by Funk et al., ${ }^{21}$ Partridge et al., ${ }^{16}$ and by Hinde. ${ }^{17}$ Funk et al. performed MP4 computations adopting a basis set derived from Huzinaga et al. ${ }^{24}$ and augmented with diffuse functions; these authors provided a well depth estimate of $4.54 \mathrm{~cm}^{-1}$ at 
TABLE I. Effect of the basis set choice on the equilibrium properties of the MgHe dimer. The different basis sets are introduced in the text. The estimate of the equilibrium parameters is obtained by interpolation with a quadratic polynomial of the energy data at $4.75,5.00$, and $5.25 \AA$.

\begin{tabular}{lcccc}
\hline \hline \multicolumn{1}{c}{$\mathrm{Mg}$} & \multicolumn{1}{c}{$\mathrm{He}$} & Bond functions & $D_{e}\left(\mathrm{~cm}^{-1}\right)$ & $r_{e}(\AA)$ \\
\hline 6-311+G3df & d-aug-cc-pVQZ & $\ldots$ & -5.21 & 5.09 \\
6-311+G3df & d-aug-cc-pV5Z & $\ldots$ & -5.36 & 5.07 \\
ANO triple zeta (Ref. 31) & d-aug-cc-pVQZ & $\ldots$ & -5.34 & 5.10 \\
ANO triple zeta & d-aug-cc-pV5Z & $\ldots$ & -5.44 & 5.08 \\
6-311+G3df & d-aug-cc-pVTZ & 332 & -5.63 & 5.07 \\
6-311+G3df & d-aug-cc-pVQZ & 332 & -5.74 & 5.07 \\
6-311+G3df & d-aug-cc-pV5Z & 332 & -5.76 & 5.06 \\
6-311+G3df & d-aug-cc-pVQZ & 33221 & -5.77 & 5.06 \\
6-311+G3df & d-aug-cc-pV5Z & 33221 & -5.78 & 5.06 \\
ANO triple zeta & d-aug-cc-pVQZ & 332 & -5.64 & 5.08 \\
ANO triple zeta & d-aug-cc-pVQZ & 33221 & -5.67 & 5.08 \\
Archibong and Thakkar (Ref. 32) & d-aug-cc-pVQZ & 332 & -5.79 & 5.06 \\
Archibong and Thakkar & d-aug-cc-pV5Z & 332 & -5.82 & 5.06 \\
Archibong and Thakkar & d-aug-cc-pVQZ & 33221 & -5.84 & 5.06 \\
\hline \hline
\end{tabular}

the $\mathrm{Mg}-\mathrm{He}$ internuclear distance of $5.16 \AA$. Partridge et al. carried out $\operatorname{CCSD}(\mathrm{T})$ computations with the aug-cc-pVQZ basis set for both $\mathrm{He}$ and $\mathrm{Mg},{ }^{25}$ supplemented with the 332 set of bond functions proposed by Tao and Pan. ${ }^{26}$ They obtained a well depth of $4.76 \mathrm{~cm}^{-1}$ at the distance of $5.09 \AA$. More recently, Hinde combined valence-only CCSDT computations of nearly full configuration-interaction (FCI) quality with a core-valence correction estimated at $\operatorname{CCSD}(\mathrm{T})$ level and, using high-quality basis sets, he proposed a minimum interaction energy of $5.01 \mathrm{~cm}^{-1}$ at the internuclear distance of $5.07 \AA$. In the present investigation, we adopted the potential given by Hinde, and we also computed two new MP4 and CCSD(T) PESs adopting a larger basis set ${ }^{21}$ and a finer spatial grid $^{16,21}$ than the available investigations with these same theoretical schemes.

Contrary to the ground-state complex, the interaction between helium and magnesium in the $\operatorname{Mg}(3 s 3 p)$ excited state has received much less attention. Indeed, to the best of our knowledge the only data available are $42 \mathrm{~cm}^{-1}$ for the well depth of the ${ }^{1} P$ state, but this single value was reported by Moriwaki and Morita ${ }^{13}$ without any details on the method of calculation. We therefore determined the complete potentialenergy curves of the three $\mathrm{Mg}(3 s 3 p)-\mathrm{He}$ states by performing multireference configuration-interaction (MRCI) computations, since these potentials are necessary for the implementation of the DIM scheme. ${ }^{18}$

All the $a b$ initio computations were carried out with the GAMESS (Ref. 27) and the GAUSSIAN (Ref. 28) suite of programs; all interaction energy data were corrected for basis set superposition error with the counterpoise scheme proposed by Boys and Bernardi. ${ }^{29}$

\section{A. Choice of the basis set}

To define an appropriate set of basis functions, we performed a series of test computations at the MP4 level in the region of the PES minimum, keeping frozen only the $1 s$ electrons of $\mathrm{Mg}$. For the $\mathrm{He}$ atom, we adopted the d-aug-cc$\mathrm{pVnZ}(n=3-5)$ sets proposed by Woon and Dunning, ${ }^{25}$ for $\mathrm{Mg}$ we considered three alternatives: the $6-311+\mathrm{G} 3 d f,^{30}$ the
Roos augmented triple-zeta atomic natural orbitals (ANO), ${ }^{31}$ and the basis set proposed by Archibong and Thakkar. ${ }^{32} \mathrm{We}$ also tested the effect of including bond functions, placed at midway between helium and magnesium, using alternatively the 332 set of Tao and $\operatorname{Pan}^{26}$ and the 33221 set by Cybulski and Toczylowski. ${ }^{33}$

The results for the combinations of the basis set considered are reported in Table I and can be summarized as follows: (i) whatever the basis set adopted for $\mathrm{Mg}$ and $\mathrm{He}$ atoms, the inclusion of bond functions lowers significantly the well depth; (ii) given a set for the two atoms, the well depths obtained with the 332 and the 33221 bond functions agree within $0.05 \mathrm{~cm}^{-1}$; (iii) once a basis set for $\mathrm{He}$ is chosen, and a set of bond functions is included, the differences among the three sets for the $\mathrm{Mg}$ atom are limited to $0.2 \mathrm{~cm}^{-1}$; and (iv) to use the d-aug-cc-pVQZ or the d-aug-cc-pV5Z set for He is nearly equivalent, provided that a set of bond functions is included. We also tested that these same conclusions hold true at the $\operatorname{CCSD}(\mathrm{T})$ level of theory. We finally devised to use the $6-311+\mathrm{G} 3 d f$ basis set for $\mathrm{Mg}$, the d-aug-cc-pVQZ set for $\mathrm{He}$, and the 332 set of bond functions, this choice representing the smallest basis set that provides interaction energies nearly converged.

\section{B. Ground-state PES}

The ground-state $\mathrm{PES}$ for $\mathrm{Mg}-\mathrm{He}$ has been computed at the MP4 and CCSD(T) levels of theory, keeping frozen only the $1 s$ electrons of magnesium. We considered 25 internuclear distances, ranging from 4.0 to $10.0 \AA$ with 0.25 - $\AA$ steps. Within this interval the energy data have been interpolated by means of natural quintic splines, whereas the longrange potential has been expressed with the analytical form $-C_{6} / r^{6}$

The computed energy curves for $\mathrm{Mg}-\mathrm{He}$ are shown in Fig. 1 along with the one proposed by $\operatorname{Hinde}^{17}$ and the He-He TTY potential. The MP4 and the CCSD(T) PESs exhibit a minimum interaction energies of -5.70 and $-4.75 \mathrm{~cm}^{-1}$, respectively, at the internuclear distances of 5.03 and $5.11 \AA$. Our CCSD(T) potential is nearly superim- 


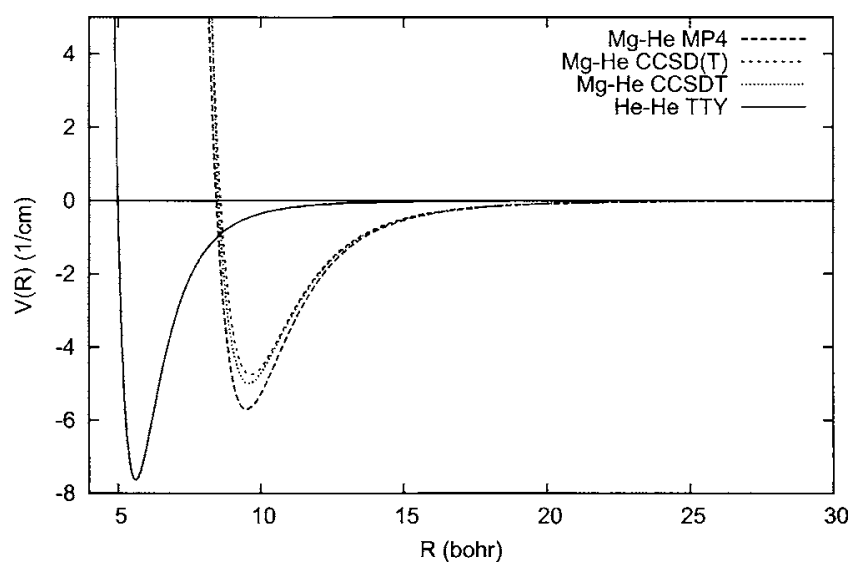

FIG. 1. Ground-state interaction energy curves for $\mathrm{Mg}-\mathrm{He}$ and $\mathrm{He}-\mathrm{He}$. The CCSDT potential is taken from Ref. 17. Energies in $\mathrm{cm}^{-1}$ and distances in a.u.

posable to the one presented by Partridge et al. ${ }^{16}$ using the same level of theory, while our MP4 estimate is significantly more attractive as compared to the one determined by Funk et $a l^{21}$ As for the potential proposed by Hinde, ${ }^{17}$ it is intermediate between $\operatorname{CCSD}(\mathrm{T})$ and $\mathrm{MP} 4$ results $(\epsilon$ $=5.01 \mathrm{~cm}^{-1}, r_{e}=5.07 \AA$ ), being much closer to the former. Finally, it is noteworthy that the computed MP4 potential is about $1 \mathrm{~cm}^{-1}$ deeper than the $\operatorname{CCSD}(\mathrm{T})$ one computed with the same basis set, contrary to the common experience for van der Waals systems. Funk et al. ${ }^{21}$ suggested that this feature is due to the quasidegenerate effects proper of the alkaline-earth metal atoms.

The gross features of these potentials allow us to draw a first picture of the behavior of $\mathrm{Mg}$ in He using the scheme proposed by Ancilotto et al. ${ }^{5}$ At variance with the uncertain situation encountered by Reho et al. ${ }^{14}$ all the new generation PESs provide the same clear-cut prediction. Indeed, the $\lambda$ values range from $2.66[\mathrm{CCSD}(\mathrm{T})$ potential] to $3.14(\mathrm{MP} 4)$, and a value of 2.78 is found for the potential by Hinde. These data are well beyond the critical value of 1.9, and even considering the correction proposed to account for the zero-point motion energy of the impurity, ${ }^{5}$ we may assert that the model unambiguously foresees the $\mathrm{Mg}$ atom to be solvated by helium.

\section{Excited-states PES}

The first singlet excited state of a free $\mathrm{Mg}$ atom is $(3 s 3 p)$ ${ }^{1} P$, and its threefold degeneracy is removed upon interaction with a He atom, thus splitting in two degenerate ${ }^{1} \Pi$ and a single ${ }^{1} \Sigma$ state. Roughly speaking, in the ${ }^{1} \Pi$ states one electron of magnesium lies in a $p$ orbital orthogonal to the $\mathrm{Mg}-\mathrm{He}$ internuclear axis, and consequently $\mathrm{He}$ essentially interacts with a positive ionic core; we expect the PES to exhibit a deep well at short distances. Conversely, in the ${ }^{1} \Sigma$ state the excited electron lies in the $p$ orbital pointing toward helium, thus generating a potential largely repulsive even at long $\mathrm{Mg}-\mathrm{He}$ distances. The PESs of these states were determined by means of CAS (four electrons in ten orbitals) computations, followed by a multireference single and double substitutions configurations interaction. The CAS scheme included in the active space the $3 s, 3 p$, and $4 s$ orbitals of $\mathrm{Mg}$,

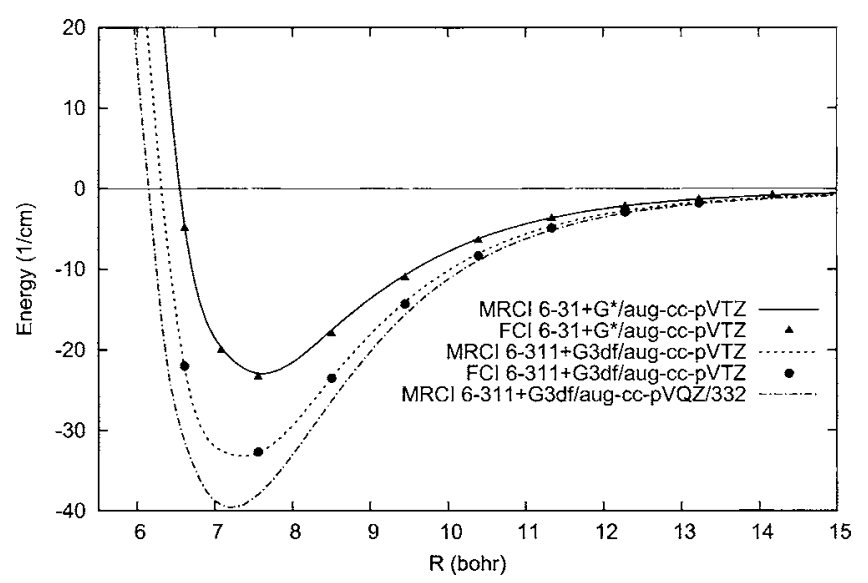

FIG. 2. ${ }^{1} \Pi$ interaction energy curve between ${ }^{1} \mathrm{P} \mathrm{Mg}$ and $\mathrm{He}$ as a function of the method (FCI or MRCI) and of the basis set used. Energies in $\mathrm{cm}^{-1}$ and distances in a.u.

and the $1 s, 2 s$, and $2 p$ orbitals of He. These computations were density averaged over the first four states.

The MRCI method is not size consistent, and therefore the interaction energies have been calculated as $E=E_{\mathrm{MgHe}}$ $-E_{\mathrm{Mg}}-E_{\mathrm{He}}+\mathrm{ESC}$, where the last term is a size consistency correction defined as $\mathrm{ESC}=E_{\mathrm{MgHe}}(r=\infty)-E_{\mathrm{Mg}}-E_{\mathrm{He}}$. To check for the soundness of this approach, we carried out an extensive comparison between MRCI and FCI computations, considering two combinations of basis sets: $6-31+\mathrm{G}^{*} / \mathrm{aug}$ -cc-pVTZ and 6-311+G3df/d-aug-cc-pVTZ, where $A / B$ indicates the set for $\mathrm{Mg}$ and $\mathrm{He}$, respectively. As it can be seen from Fig. 2 and 3, the overall agreement between MRCI and FCI results is very satisfactory for the two basis set choices. We are therefore confident that even the use of a more extended basis set would not alter this agreement, and thus we determined the three PESs at MRCI level using the same basis set as for the ground-state case. The interaction energies have been determined on $26 \mathrm{Mg}-\mathrm{He}$ arrangements, ranging from 3.0 to $12.0 \AA$; the analytical representations are obtained according to the same strategy adopted for the ground state. The ${ }^{1} \Pi$ potential reported in Fig. 2 exhibits a well depth of $39.58 \mathrm{~cm}^{-1}$ at the equilibrium distance of $3.82 \AA$, whereas the ${ }^{1} \Sigma$ curve is repulsive from $7.79 \AA$ in-

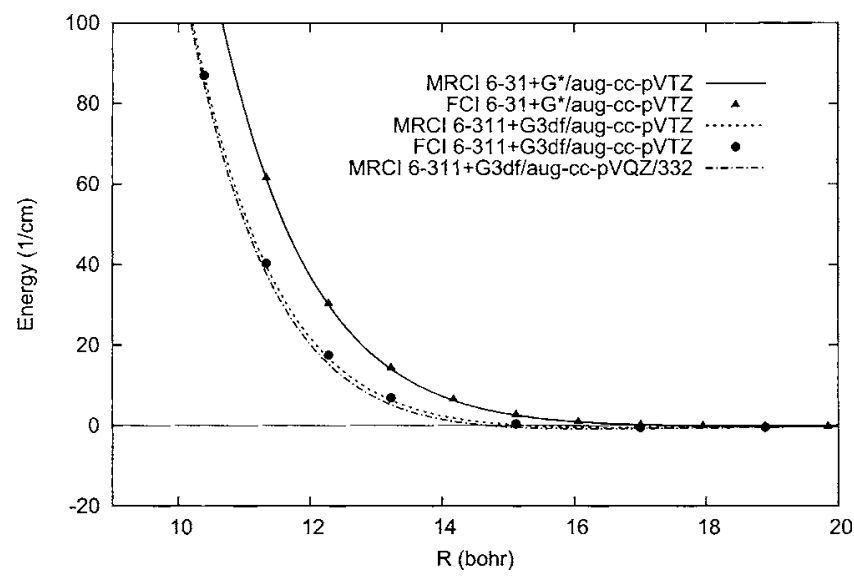

FIG. 3. ${ }^{1} \Sigma$ interaction energy curve between ${ }^{1} P \mathrm{Mg}$ and $\mathrm{He}$ as a function of the method (FCI or MRCI) and of the basis set used. Energies in $\mathrm{cm}^{-1}$ and distances in a.u. 


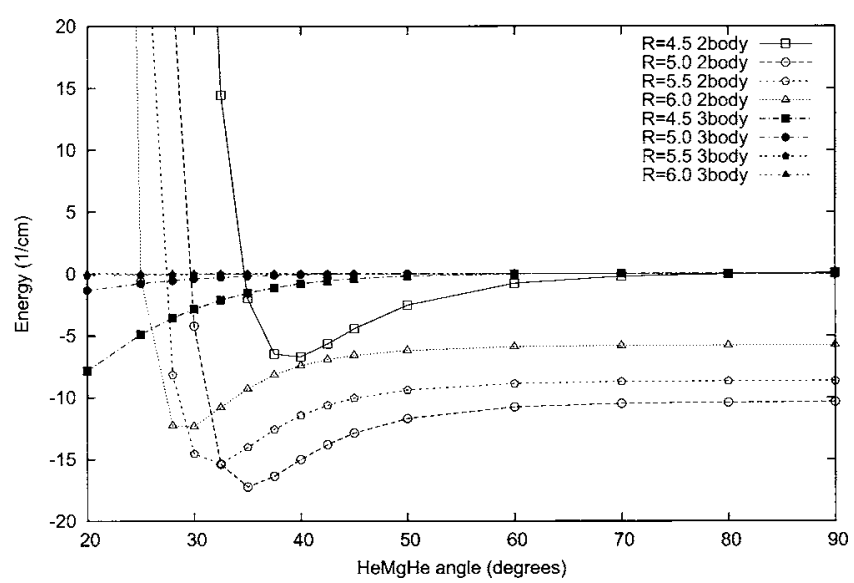

FIG. 4. Two-body (empty symbols) and three-body (filled symbols) effects computed at the MP4 level for $\mathrm{MgHe}_{2}$ complexes in isosceles triangular geometry, as a function of the $\mathrm{MgHe}$ distance and the HeMgHe angle. Energies in $\mathrm{cm}^{-1}$, distances in angstroms and angles in degrees.

ward, and has a very shallow well of $0.81 \mathrm{~cm}^{-1}$ at $8.62 \AA$, as reported in Fig. 3. Finally, the asymptotic separation between the excited levels and the ground state was set to the experimental value of $35051.264 \mathrm{~cm}^{-1}$. ${ }^{34}$

\section{Relevance of the many-body contributions}

As mentioned in the Introduction, in the DMC simulations the ground-state PES of $\mathrm{MgHe}_{n}$ has been approximated as a sum of pairwise interactions. Among the contributions excluded, the major role is certainly played by the threebody effects. In systems scarcely polarizable such as the helium aggregates, the most important three-body component arises from the nonadditivity of both the exchange and dispersion terms, and the former is generally much larger than the latter. Exchange contributions are typically attractive in triangular arrangements and repulsive in collinear geometries, while the dispersion ones behave oppositely. In helium-based systems these effects are usually quite small, and therefore the common practice is to neglect them regardless of the doping impurity.

Nevertheless, we carried out a series of test computations to check the validity of this approximation in $\mathrm{Mg}$ doped He clusters. We considered $\mathrm{MgHe}_{2}$ complexes with $\mathrm{C} 2 v$ symmetry, since the highest three-body contributions arise in triangular arrangements. Moreover, this choice reduces the calculation of the three-body contributions to the expression

$$
\begin{aligned}
\Delta E(3 \text { body })= & E\left(\mathrm{MgHe}_{2}\right)-2 E(\mathrm{MgHe})-E\left(\mathrm{He}_{2}\right) \\
& +E(\mathrm{Mg})+2 E(\mathrm{He})
\end{aligned}
$$

that requires five energy values for each geometry. All the terms in the right-hand side of Eq. (2) were computed at the MP4 level of theory, adopting always the complete trimercentered basis set. We adopted the same atomic basis functions as for the two-body potentials, but for the exclusion of the bond functions, that proved nearly negligible in this respect. In Fig. 4 we report the total two- and three-body contributions to the interaction energy as scans along the $\mathrm{HeMgHe}$ angle at fixed $\mathrm{Mg}-\mathrm{He}$ internuclear distances. The three-body terms are not negligible only at short $\mathrm{Mg}-\mathrm{He}$ distances and small $\mathrm{He}-\mathrm{Mg}-\mathrm{He}$ angles. In these arrangements the two-body PES is only slightly attractive or even repulsive, and therefore we are confident that the inclusion of many-body effects in the $\mathrm{MgHe}_{n}$ complexes would scarcely affect the results of the DMC simulations.

\section{METHODS}

It is well known that pure and doped He clusters are characterized by a highly quantum nature, a feature manifesting itself in a small total binding energy and a wide anharmonic motion of both the doping impurity and the He atoms. As a consequence of the intrinsic anharmonicity and of the experimental size of these clusters (usually of the order of several thousands of atoms), the possibility of using either the harmonic approximation or more accurate basis set/gridbases approaches is usually hindered.

To describe at atomistic level the solvation properties of doped clusters and to compute their excitation spectra, we believe that the QMC methods are the best-suited techniques. Since these methods are well described in the literature, ${ }^{35}$ we restrain ourselves from presenting long discussions, except for the technical details that are relevant to the present work. Here, we employed variational Monte Carlo (VMC) to optimize a trial wave function $\Psi_{T}(\mathbf{R})$ and DMC to correct the remaining deficiencies of $\Psi_{T}(\mathbf{R})$, projecting out all the excited-state components and sampling $f(\mathbf{R})=\Phi_{0}(\mathbf{R}) \Psi_{T}(\mathbf{R})$, where $\Phi_{0}(\mathbf{R})$ is the exact ground-state wave function for the system. In both cases a description of the ground state is sought, the low temperature of the clusters $(0.37 \mathrm{~K})$ and the large energy gap between vibrational excited states in $\mathrm{He}_{n}$, suggesting that thermal excitations should not play a relevant role (for a discussion on this topic see Ref. 7).

In a.u., the Hamiltonian operator for $\mathrm{MgHe}_{n}$ reads as

$$
\mathcal{H}=-\frac{1}{2}\left(\sum_{i=1}^{n} \frac{\nabla_{i}^{2}}{m^{4} \mathrm{He}}+\frac{\nabla_{\mathrm{Mg}}^{2}}{m_{\mathrm{Mg}}}\right)+V(\mathbf{R}) \text {. }
$$

As mentioned before, we assume a potential of the form $V(\mathbf{R})=\sum_{i<j} V_{\mathrm{HeHe}}\left(r_{i j}\right)+\sum_{i} V_{\mathrm{MgHe}}\left(r_{i \mathrm{Mg}}\right)$ for the clusters with the magnesium atom in the ${ }^{1} S$ electronic ground state.

Our trial wave function has the common form

$$
\Psi_{T}(\mathbf{R})=\prod_{i<j}^{N} \psi\left(r_{i j}\right) \prod_{i}^{N} \phi\left(r_{i \mathrm{Mg}}\right),
$$

where no one-body part was used, and ${ }^{36}$

$$
\psi(r)=\phi(r)=\exp \left[-\frac{p_{5}}{r^{5}}-\frac{p_{2}}{r^{2}}-p_{1} r-p_{0} \ln (r)\right] .
$$

The parameters of the model wave function were fully optimized minimizing the mean absolute deviation of the local energy $E_{\text {loc }}(\mathbf{R})=\mathcal{H} \Psi_{T}(\mathbf{R}) / \Psi_{T}(\mathbf{R})=\mathcal{H}_{\text {loc }}(\mathbf{R})$ over a fixed set of points (roughly 5000), as proposed in Ref. 37. The optimized wave functions were successively employed to guide the DMC simulations of the doped clusters in order to sample the mixed distribution $f(\mathbf{R})=\Phi_{0}(\mathbf{R}) \Psi_{T}(\mathbf{R})$. The simulations were carried out using the standard drift-diffusebranch scheme with an acceptance-rejection step, ${ }^{35}$ a time 
TABLE II. DMC energy $E_{\mathrm{DMC}}(n)$ and $\Delta(n)=[E(n)-E(m)] /(n-m)$ as a function of the number of He atoms in the cluster for the three interaction potentials. For a given $n, \mathrm{MgHe}_{m}$ is the largest cluster available with $n$ $>m$. Energetic quantities are in $\mathrm{cm}^{-1}$.

\begin{tabular}{ccccccc}
\hline \hline$n$ & $E_{\mathrm{DMC}}^{\mathrm{MP} 4}$ & $\Delta_{\mathrm{DMC}}^{\mathrm{MP} 4}$ & $E_{\mathrm{DMC}}^{\mathrm{CCSD}(\mathrm{T})}$ & $\Delta_{\mathrm{DMC}}^{\mathrm{CCSD}(\mathrm{T})}$ & $E_{\mathrm{DMC}}^{\mathrm{CCSDT}}$ & $\Delta_{\mathrm{DMC}}^{\mathrm{CCSDT}}$ \\
\hline 2 & $-2.5366(6)$ & $-1.2683(3)$ & & & & \\
4 & $-5.660(2)$ & $-1.5618(8)$ & $-4.127(4)$ & $-1.0317(4)$ & & \\
6 & $-9.2445(9)$ & $-1.7921(9)$ & $-6.9881(9)$ & $-1.431(1)$ & & \\
8 & $-13.172(5)$ & $-1.964(2)$ & $-10.244(5)$ & $-1.628(3)$ & & \\
12 & $-21.795(3)$ & $-2.156(1)$ & $-17.619(6)$ & $-1.843(2)$ & $-18.406(4)$ & \\
15 & $-28.84(1)$ & $-2.346(3)$ & $-23.717(3)$ & $-2.032(2)$ & $-24.648(7)$ & $-2.081(4)$ \\
18 & $-36.33(1)$ & $-2.498(5)$ & $-30.26(2)$ & $-2.182(7)$ & $-31.26(1)$ & $-2.204(3)$ \\
20 & $-41.54(2)$ & $-2.61(1)$ & $-34.76(2)$ & $-2.25(1)$ & $-35.894(7)$ & $-2.317(5)$ \\
25 & $-55.031(8)$ & $-2.698(4)$ & $-46.73(2)$ & $-2.394(5)$ & $-47.984(9)$ & $-2.418(2)$ \\
30 & $-68.75(3)$ & $-2.744(6)$ & $-59.37(3)$ & $-2.526(7)$ & $-60.75(1)$ & $-2.553(2)$ \\
40 & $-97.13(5)$ & $-2.838(6)$ & $-85.88(3)$ & $-2.651(4)$ & $-87.93(2)$ & $-2.718(2)$ \\
50 & $-126.70(9)$ & $-2.96(1)$ & $-114.27(5)$ & $-2.839(5)$ & $-116.84(3)$ & $-2.891(4)$ \\
\hline \hline
\end{tabular}

step of 100 hartree $^{-1}$, and a target population of roughly 5000 walkers. The distributions $f(\mathbf{R})$ were used to compute exactly the energy values using the mixed estimator

$$
\langle\mathcal{H}\rangle_{M}=\frac{\int f(\mathbf{R}) \mathcal{H}_{\mathrm{loc}}(\mathbf{R}) d \mathbf{R}}{\int f(\mathbf{R}) d \mathbf{R}}
$$

as well as the mixed and second-order estimate (SOE) $\langle\mathcal{O}\rangle_{\mathrm{SOE}}=2\langle\mathcal{O}\rangle_{M}-\langle\mathcal{O}\rangle_{\mathrm{VMC}}$ of many other expectation values (e.g., the interparticle distribution functions). The SOE was used to reduce the bias introduced in the mixed estimate of operators that do not commute with the Hamiltonian by the use of a nonexact trial wave function.

As for the absorption spectrum of the $\mathrm{Mg}$ atom, we computed it with the same semiclassical approach used in Ref. 7 to compute the Ag spectrum, adapting a technique previously proposed by Cheng and Whaley ${ }^{38}$ for the Franck-Condon line shapes of an electronic transition in a condensed phase system. The method was originally presented by $\mathrm{Lax}^{39}$ and was modified to take into account the system temperature of $0 \mathrm{~K}$. In its crudest approximation, the spectral lines of a chromophore are computed collecting the distribution of the differences $V_{\text {exc }}(\mathbf{R})-V_{\mathrm{gs}}(\mathbf{R})$ over the sampled $f(\mathbf{R})$. In our case, $V_{\mathrm{gs}}(\mathbf{R})\left[V_{\text {exc }}(\mathbf{R})\right]$ is the interaction potential between the ground- (excited- ) state $\mathrm{Mg}$ atom with the He atoms. The three electronic states for the excited $\mathrm{Mg}$ attached to the $\mathrm{He}$ cluster are obtained from the two dimer excited potentials ${ }^{1} \Sigma$ and ${ }^{1} \Pi$ using the DIM method. ${ }^{18}$ Since all the details needed to implement it are well described by Nakayama and Yamashita, ${ }^{8}$ we refer to their paper and to Ref. 7 for further discussions, especially related to the general accuracy of the method.

\section{RESULTS}

\section{A. Energetics}

Due to the highly quantum nature of $\mathrm{He}_{n}$, we limit our presentation of the $\mathrm{MgHe}_{n}$ energetics only to the fully quan- tal DMC results and postpone a short discussion on the potential-energy values of the global minimum structures in Sec. IV B

The DMC energies $E(n)$ and the differential values $\Delta(n)=[E(n)-E(m)] /(n-m)$ obtained using the three potentials for the $\mathrm{MgHe}_{n}$ clusters are presented in Table II. Here, $\mathrm{MgHe}_{m}$ is the largest cluster for which $n>m$ still holds. From these energetic results, it appears that the $\operatorname{CCSD}(\mathrm{T})$ and the CCSDT data give a very similar description of the clusters, while the MP4 produces slightly lower total energies as expected on the basis of the deeper well.

The $\Delta(n)$ values can be interpreted as the negative of the He evaporation energies and are shown in Fig. 5. From this, we notice that for all three interaction potentials $\Delta(n)$ monotonically decrease upon increasing $n$, a finding that is different from what is usually found for impurities interacting strongly with $\mathrm{He},{ }^{7,40,41}$ and for which a multiple-shell effect is usually found. Instead, the behavior of $\Delta(n)$ for $\mathrm{MgHe}_{n}$ is quite similar to the one obtained for pure $\mathrm{He}_{n}$ (Ref. 42) (also shown in Fig. 5) and for dopants floating on the surface., ${ }^{9,11}$ In particular, our $\operatorname{CCSD}(\mathrm{T})$ and $\operatorname{CCSDT} \Delta(n)$ values for the large clusters are very close to the pure cluster results presented in Ref. 42. The MP4 potential gives somewhat larger

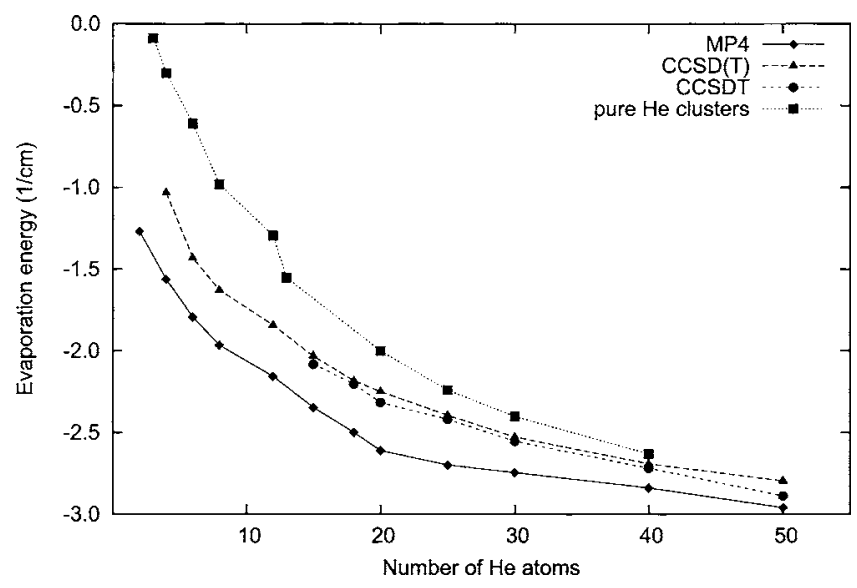

FIG. 5. Evaporation energy $\Delta(n)$ as a function of the interaction potential and of the number of helium atoms for $\mathrm{MgHe}_{n}$. The $\Delta(n)$ values for pure $\mathrm{He}_{n}$ are also included. Energies in $\mathrm{cm}^{-1}$. 
TABLE III. Binding energy of $\mathrm{Mg}$ to $\mathrm{He}_{n}$ as a function of the number $n$ of $\mathrm{He}$ atoms in the cluster for the three interaction potentials. Quantities are in $\mathrm{cm}^{-1}$.

\begin{tabular}{cccc}
\hline \hline$n$ & $E_{\mathrm{DMC}}^{\mathrm{MP} 4}$ & $E_{\mathrm{DMC}}^{\mathrm{CCSD}(\mathrm{T})}$ & $E_{\mathrm{DMC}}^{\mathrm{CCSDT}}$ \\
\hline 2 & $2.5357(6)$ & & \\
4 & $5.271(2)$ & $3.738(4)$ & \\
6 & $7.6368(9)$ & $5.3804(9)$ & \\
8 & $9.604(5)$ & $6.676(5)$ & \\
12 & $13.049(7)$ & $8.873(6)$ & $9.660(7)$ \\
20 & $18.50(2)$ & $11.72(2)$ & $12.85(1)$ \\
25 & $24.4(3)$ & $16.1(3)$ & $17.4(3)$ \\
30 & $26.69(3)$ & $17.31(3)$ & $18.69(3)$ \\
40 & $26.85(5)$ & $15.60(3)$ & $17.65(3)$ \\
\hline \hline
\end{tabular}

values for the same quantity, a difference in touch with the slightly larger interaction energy computed by the MP4 method and that might induce a more compact structure for the He moiety.

Another interesting quantity is represented by the binding energy $(\mathrm{BE})$ of $\mathrm{Mg}$ to the helium cluster $\mathrm{BE}(n)=E^{\mathrm{He}_{n}}$ $-E^{\mathrm{MgHe}_{n}}$ (often indicated as the dopant chemical potential) shown in Table III for $\mathrm{He}_{n}$ up to $n=40$. These values were obtained using the DMC energies for the pure helium clusters presented in Ref. 7. The energy data obtained with the MP4 potential monotonically increase with cluster size, and appear to be nearly converged at $n=40$. A similar behavior is found using the CCSDT and $\operatorname{CCSD}(\mathrm{T})$ potentials up to $n$ $=30$, but in these cases there is an unexpected decrease of $\mathrm{BE}$ when the largest clusters $(n=40)$ are considered. This different behavior of $\mathrm{BE}$ is due to relevant differences in the structures of the doped clusters depending on the potential adopted and the cluster size, as will be discussed in Sec. IV B.

\section{B. Structure}

Let us start considering the relative shape of the $\mathrm{MgHe}$ and $\mathrm{HeHe}$ interaction curves shown in Fig. 1. It is clearly evident that the $\mathrm{He}-\mathrm{He}$ curve has a deeper minimum $\epsilon$ and a shorter equilibrium distance $r_{e}$ than all the $\mathrm{Mg}-\mathrm{He}$ ones. Similar situations have been previously investigated ${ }^{43}$ and it is easy to predict that the largest species should segregate on the surface to reduce the strain in the optimized structure. It is therefore quite likely that a classical minimization of the cluster interaction potential would produce global minimum structures showing a compact He moiety with Mg lying outside this core, as already found for $\mathrm{Ne}_{n} \mathrm{H}^{-}$(Ref. 44) and $\mathrm{He}_{n} \mathrm{H}^{-} .{ }^{11}$ To confirm this tendency for our systems, we minimized the interaction potential starting from several thousands of random configurations for each cluster, invariably obtaining a "floating" Mg impurity and a compact He moiety as lowest-energy structure. As a consequence, the solubility of $\mathrm{Mg}$ in $\mathrm{He}_{n}$ suggested by the cluster experiments ${ }^{14}$ must be considered a purely quantum phenomenon.

To extract the structural properties of the $\mathrm{Mg}$-doped clusters from DMC simulations, we computed several average values and distribution functions for the particle-particle

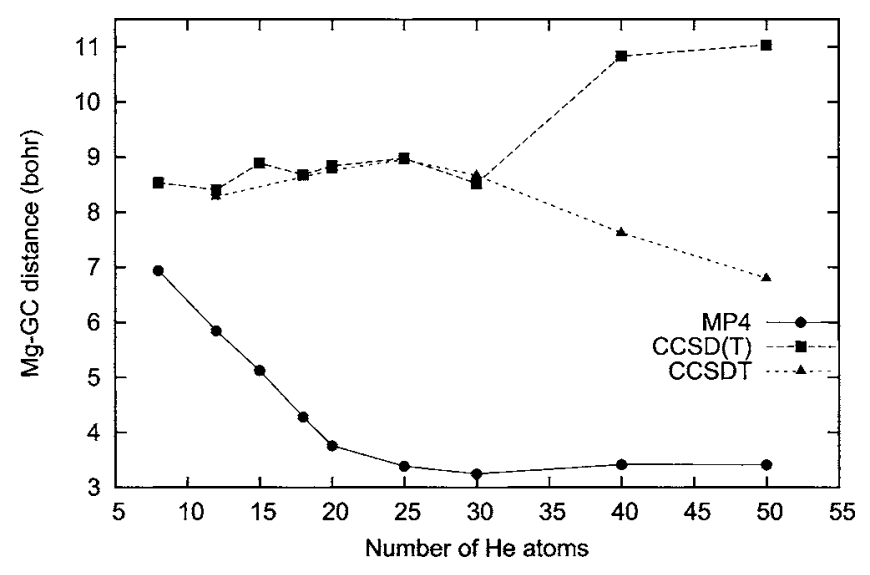

FIG. 6. Average distance (bohr) between $\mathrm{Mg}$ and the geometrical center (gc) of the He moiety as a function of the number of $\mathrm{He}$ atoms in the cluster and of the interaction potential.

distances and for the distance between a particle $A$ (either $\mathrm{He}$ or $\mathrm{Mg}$ ) and the (geometrical) center $(\mathrm{gc})$ of the He moiety

$$
\begin{aligned}
R_{\mathrm{gcMg}} & =\left\|\mathbf{r}_{\mathrm{Mg}}-\sum_{i=1}^{n} \mathbf{r}_{i} / n\right\|, \\
R_{\mathrm{gcHe}} & =\left\|\sum_{j}^{n}\left(\mathbf{r}_{j}-n^{-1} \sum_{i=1}^{n} \mathbf{r}_{i}\right) / n\right\| .
\end{aligned}
$$

Figure 6 shows the behavior of the average $R_{\mathrm{gcMg}}$ as a function of the number of $\mathrm{He}$ atoms in the cluster for the three interaction potentials used in this work. As for the MP4 potential, $\left\langle R_{\mathrm{gcMg}}\right\rangle$ shows a steep decrease upon increasing $n$, strongly resembling the case of $\mathrm{AgHe}_{n}$ (Ref. 7) where this behavior indicated the onset of solvation of the $\mathrm{Ag}$ atom in the He clusters. Conversely, the two CC-based pair interactions produced somehow unexpected and peculiar behaviors that, to the best of our knowledge, have never been found before with other dopants. More precisely, both the $\operatorname{CCSD}(\mathrm{T})$ and CCSDT potentials generated almost constant $\left\langle R_{\mathrm{gcMg}}\right\rangle$ values for $n \leqslant 30$; this similar behavior is then followed by a sudden increase for $\operatorname{CCSD}(\mathrm{T})$ and a less rapid decrease in the case of CCSDT. Indeed, the trends shown by both $\operatorname{CCSD}(\mathrm{T})$ and CCSDT are somewhat intermediate between the one exhibited by the impurities undergoing solvation (with $R_{\mathrm{gcMg}}$ decreasing as a function of $n$ ) and the ones floating on the helium droplet surface (in these systems $R_{\mathrm{gcMg}}$ monotonically increases with increasing $n$ ) as in the case of $\mathrm{H}^{-} \mathrm{He}_{n}{ }^{9,11}$ These unusual features are hardly interpreted on the basis of the available literature results, and to put them in relation with a definite cluster structure-particularly concerning the onset of solvation-requires a more detailed treatment of the DMC data.

Figure 7 shows the pair distribution function $p\left(R_{\mathrm{gcMg}}\right)$ for several $\mathrm{MgHe}_{n}$ clusters and for any of the PES adopted: MP4, CCSD(T), and CCSDT. The curves in the figure are normalized so that $4 \pi \int_{0}^{\infty} r^{2} p(r) d r=1$. As clearly seen in Fig. 7(a), the simulations with the MP4 potential produced a solvated dopant sitting at the center of the large He clusters, the smaller ones showing a slightly different behavior due to the incomplete first solvation shell of Mg. The simulations car- 


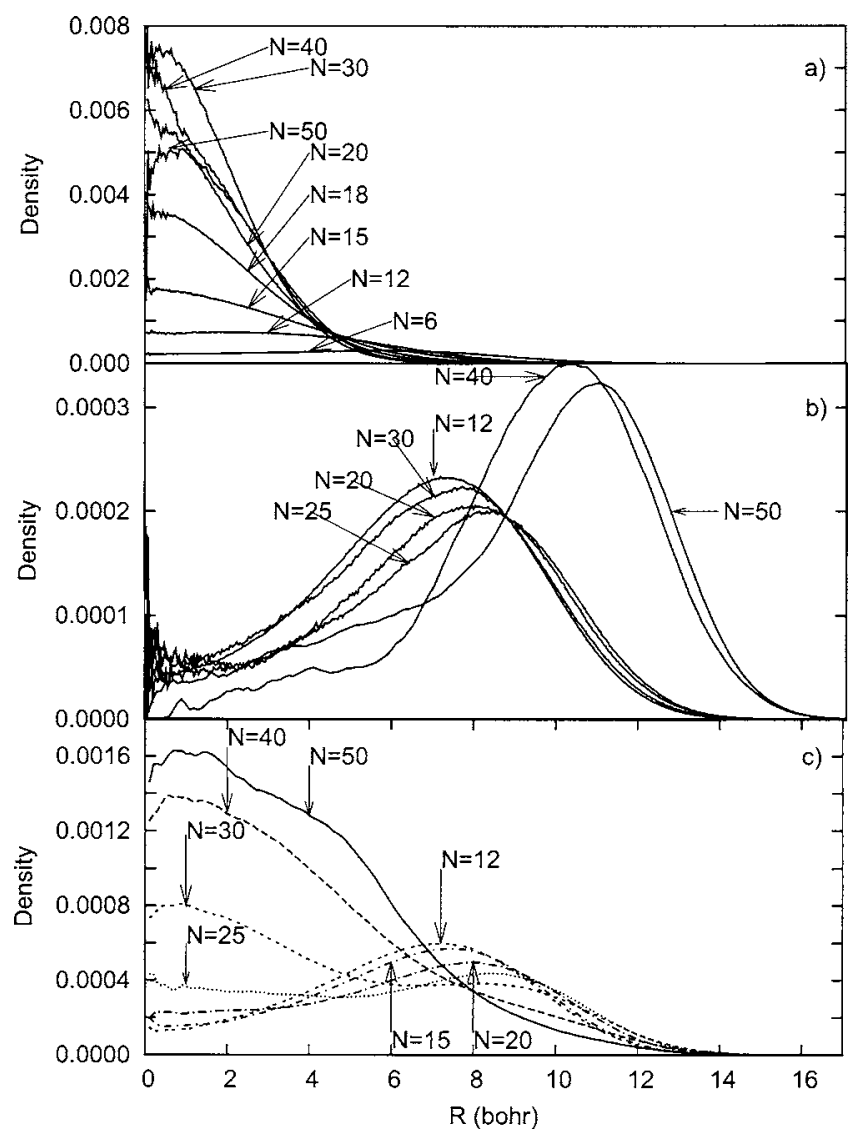

FIG. 7. Mg radial probability density function with respect to the gc of the He moiety as a function of the number of He atoms in the cluster and of the interaction potential: (a) MP4, (b) $\operatorname{CCSD}(\mathrm{T}$ ), and (c) CCSDT. Distances in bohrs.

ried out using the $\operatorname{CCSD}(\mathrm{T})$ potential [Fig. 7(b)] gave instead a largely different prediction of the solvation properties: for $n \leqslant 30$, the distributions present a peak with the maximum in the range of 7-8 bohr, therefore locating $\mathrm{Mg}$ relatively far away from the center of the droplet and explaining the almost constant $R_{\mathrm{gcMg}}$ values; for $n=40$ and 50, the peak maximum is displaced further away from the He moiety center, clearly indicating the lack of solvation for $\mathrm{Mg}$. As for the CCSDT results [Fig. 7(c)], the behavior of $p\left(R_{\mathrm{gcMg}}\right)$ is even more complicated. For $n \leqslant 20$, the distributions show a single peak whose maximum is located in the range of 7-8 bohr similar to the $\operatorname{CCSD}(\mathrm{T})$ ones. Upon increasing $n$, they first assume a bimodal shape $(n=25$ and 30$)$, and then transform their shape building a single broad peak centered at $R_{\mathrm{gcMg}}=0$.

As mentioned before, the dependency of the solvation properties on the number of $\mathrm{He}$ atoms shown by both the $\operatorname{CCSD}(\mathrm{T})$ and CCSDT $\mathrm{Mg}-\mathrm{He}$ pair interactions has never been observed for any previously studied dopant. In the CCSDT case, our findings suggest the onset of a dynamical many-body effect stabilizing the solvated dopant more than the surface one after a critical number of helium is reached. A likely explanation for this behavior is obtained considering what happens displacing a $\mathrm{Mg}$ atom from the surface to the interior of the droplet. During this process, a cavity is formed inside the cluster due to the incoming $\mathrm{Mg}$, this cavity and the displaced He atoms contribute to increase the droplet exter-

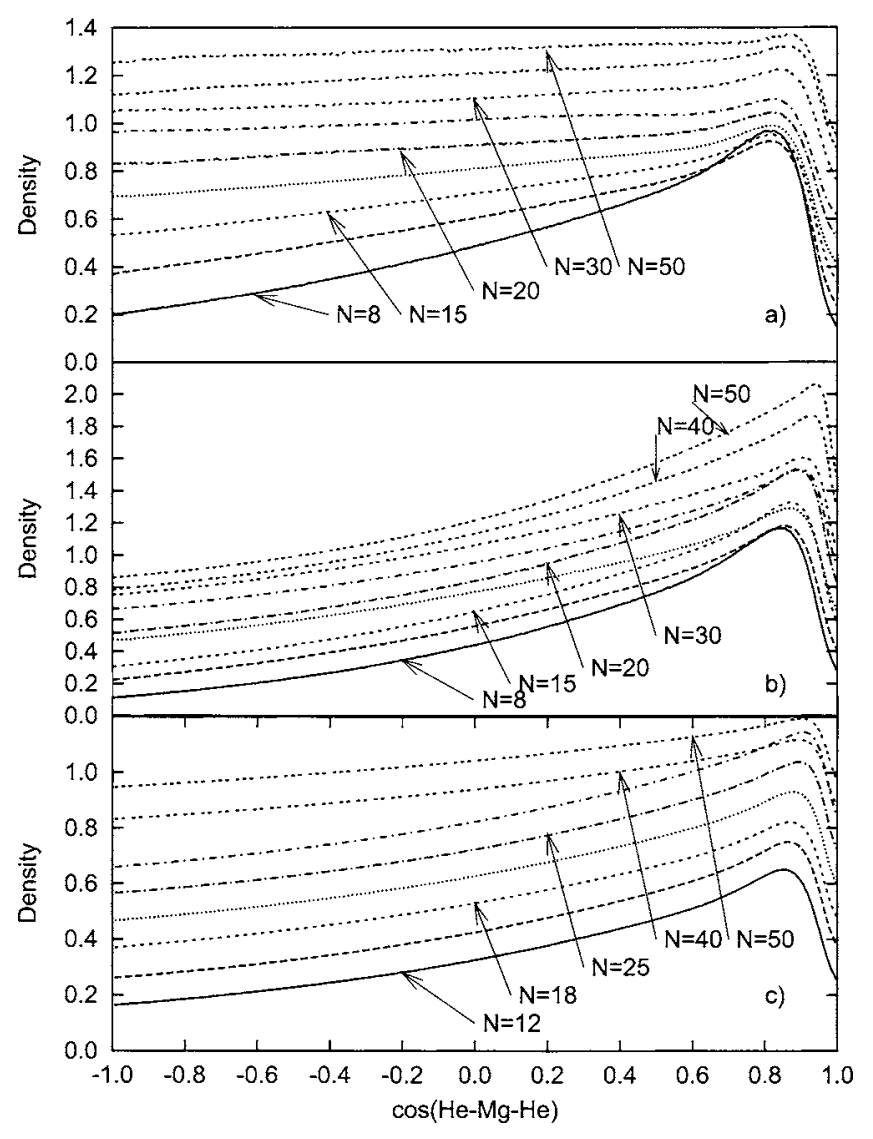

FIG. 8. Probability density function for the cosine of the $\mathrm{He}-\mathrm{Mg}-\mathrm{He}$ angle as a function of the number of He atoms in the cluster and of the interaction potential: (a) MP4, (b) CCSD(T), and (c) CCSDT. Each curve has been shifted upward by 0.1 with respect to the previous one to facilitate the comparison.

nal surface area by a quantity $\Delta S$. Upon increasing $n$ (and hence the total volume of the He moiety), the absolute value of $\Delta S$ is reduced generating, as a consequence, a smaller increase in "surface energy" due to the lost He-He interactions, and making more favorable the dopant solvation. ${ }^{45}$ It must be stressed, however, that the dopant solubility depends also on the shape (e.g., well depth and location) of the interaction energy between $\mathrm{He}$ and $\mathrm{Mg}$; if the energy loss due to the formation of the cavity and the increase in surface area is not overcompensated by the stronger interaction between the solvated dopant and the droplet, the impurity will be segregated on the surface as a way to reduce the total energy of the system. So, the cause for the different behavior of the $\operatorname{CCSD}(\mathrm{T})$ and CCSDT distributions must be sought in the slight differences presented by the two interaction potentials.

A somehow different perspective of the structural features of $\mathrm{MgHe}_{n}$ is given by the distributions of the cosine values for the HeMgHe angle presented in Fig. 8 where, again, different behaviors are shown. In the case of the MP4 interaction [Fig. 8(a)], the distributions show a gradual change upon increasing the number of He atoms; the pronounced features present in the distribution for the small clusters [i.e., the sharp maximum around $\cos (\mathrm{HeMgHe})$ $=0.8$ and the two minima at $\cos (\mathrm{HeMgHe})=1$ and -1$]$ smooth for larger $n$ producing, in the case of $\mathrm{MgHe}_{50}$, a substantial raise of the minimum at $\cos (\mathrm{HeMgHe})=1$, a dis- 
placed short maximum around $\cos (\mathrm{HeMgHe})=0.85$, and a plateau at lower values of the cosine. A similar behavior was exhibited by the $\mathrm{AgHe}_{n}$ clusters, ${ }^{7}$ an evidence of the complete solvation of the dopant and of the formation of a compact and ordered first shell of $\mathrm{He}$ atoms around the silver atom. Furthermore, the raise of the minimum at $\cos (\mathrm{HeAgHe})=1$ indicated the onset of the second solvation shell for Ag. The same process occurs also for $\mathrm{MgHe}_{n}$ when the MP4 data are considered, though a substantial difference between $\mathrm{Mg}$ - and Ag-doped clusters occurs. Indeed, in $\mathrm{AgHe}_{n}$ the plateau at low cosine values is present even in small clusters (e.g., $\mathrm{AgHe}_{6}$ ), while in $\mathrm{MgHe}_{n}$ it becomes evident only for clusters with at least $25 \mathrm{He}$ atoms. This finding is probably a consequence of the smaller $\epsilon$ and the larger $r_{e}$ in the $\mathrm{MgHe}$ interaction potential than in the $\mathrm{AgHe}$ one, the shallower interaction curve allowing the $\mathrm{He}$ atoms in $\mathrm{MgHe}_{n}$ to be less tightly bound to the dopant and to cluster together in a more compact way. In turn, this retards the formation of an angularly uniform first solvation shell around the dopant.

Figure 8(b) shows the cosine distribution functions for the $\operatorname{CCSD}(\mathrm{T})$ curve, two striking differences being apparent with respect to the previous results. First, only small changes in the distributions are produced upon increasing the number of He atoms; this indicates the presence of a similar structure for all the clusters. Second, the density at $\cos (\mathrm{HeMgHe})=1$ is substantially different from zero already at $n=8$ and increases for larger $n$; this feature suggests that two He atoms can be found along the same radius departing from $\mathrm{Mg}$ even in the small clusters, and these angular distributions would be consistent with a dopant floating on the surface of the $\mathrm{He}$ droplet whose radius increases upon increasing the number of He atoms. At variance with this interpretation, Fig. 8(b) also shows a substantial density at $\cos (\mathrm{HeMgHe})=-1$ for the small clusters suggesting the presence of a less "clear-cut" structural situation that can be explained either by imaging the $\mathrm{Mg}$ atom as "sitting" in a deep dimple on the surface and having a fluctuating "crown" of He atoms in equatorial position or, alternatively, by invoking large excursions of one helium away from the cluster surface and around the dopant.

Figure 8(c) shows the probability densities obtained during the DMC simulations employing the CCSDT pair interaction. For $n \leqslant 30$, these distributions show an almost identical shape and are quite similar to the ones obtained using the $\operatorname{CCSD}(\mathrm{T})$ curve, therefore indicating the presence of a strongly anisotropic environment around the $\mathrm{Mg}$ atom. Upon increasing $n$, the distributions change smoothing the maximum around $\cos (\mathrm{HeMgHe})=0.9$ and increasing the density at lower values of $\cos (\mathrm{HeMgHe})$, a finding which is in phase with the behavior of the radial probability density previously described. However, the absence of the distribution plateau characterizing the MP4 results at low cosine indicates that the $\mathrm{He}$ environment around $\mathrm{Mg}$ is still far from being isotropic.

The overall picture given by the results discussed in this section is very intriguing. First, we found that even very small differences among the potentials adopted in the DMC simulations produce quite different structures in the doped clusters. Second, the onset of the solvation itself is highly sensitive to the very small changes in the interaction poten-

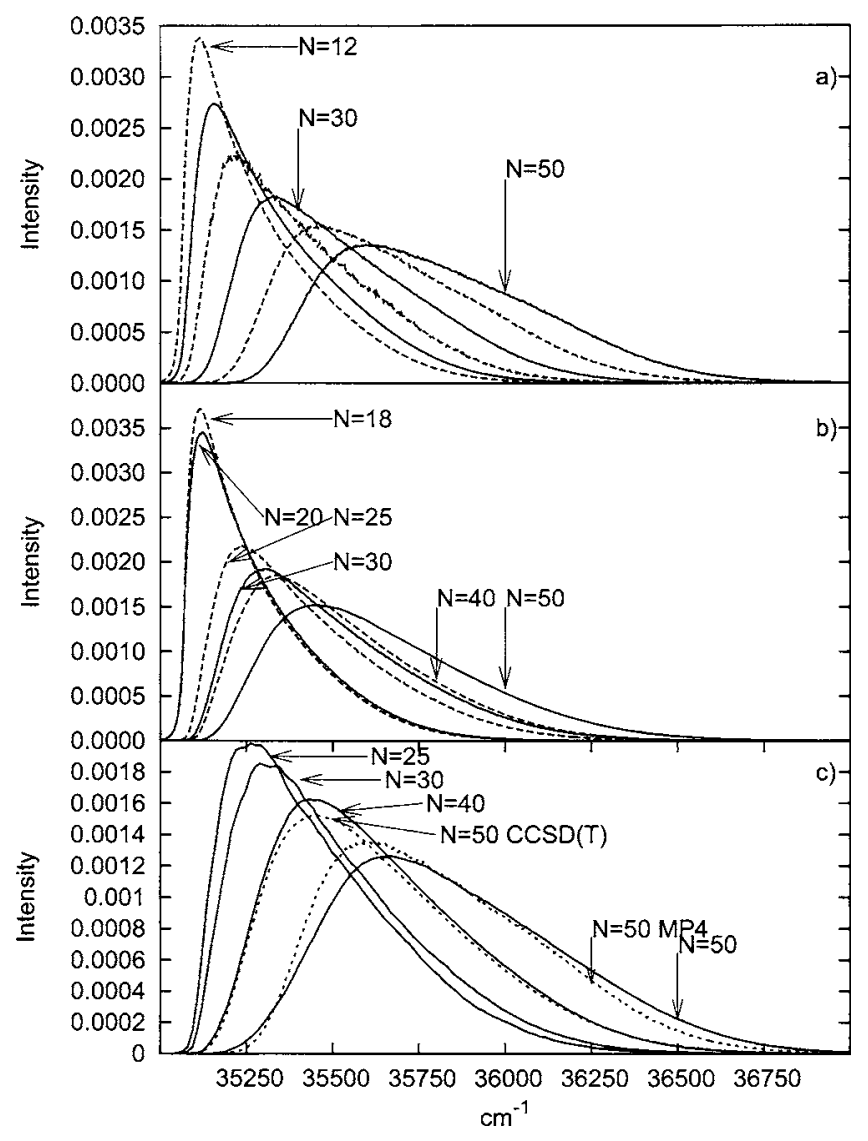

FIG. 9. Simulated excitation spectra of $\mathrm{Mg}$ attached to $\mathrm{He}_{n}$ clusters. (a) Results obtained using the MP4 potential for $n=12,15,20,30,40$, and 50. (b) Results obtained using the $\operatorname{CCSD}(\mathrm{T})$ potential for $n=18,20,25,30,40$, and 50. (c) Results obtained using the CCSDT potential for $n=25,30,40$, and 50. The results obtained with MP4 and $\operatorname{CCSD}(\mathrm{T})$ for $\mathrm{MgHe}_{50}$ are also reported in this panel for a direct comparison.

tial of the dopant and to the number of He atoms contained in the droplet. In borderline systems it is therefore quite restrictive to describe the solvation as simply occurring or not, and the properties of the dopant are very hard to classify [see the DMC simulations on $\mathrm{MgHe}_{n}$ adopting the CCSD(T) and CCSDT PESs]. Further discussion on the role of the $\mathrm{Mg}-\mathrm{He}$ interaction energy curve in several components of the solvation phenomenon is outlined in Sec. IV D.

\section{Excitation spectra of $\mathrm{Mg}$ in $\mathrm{He}_{n}$}

As mentioned in the Introduction, the experimental spectra for the ${ }^{1} P \leftarrow{ }^{1} S$ excitation of $\mathrm{Mg}$ dispersed in superfluid bulk $\mathrm{He}$ (Ref. 13) and attached to He nanodroplets ${ }^{14}$ are available. Their features, as well as the comparison with excitation spectra of the free $\mathrm{Mg}$ atom, strongly suggest that $\mathrm{Mg}$ is soluble in superfluid helium. In this section, we discuss the simulation results obtained employing the various ground-state interaction curves for $\mathrm{MgHe}$ as a function of the number of helium atoms, and compare the experimental spectra with the simulated ones for the largest clusters available.

Figures 9(a)-9(c) show the spectra computed with the MP4, CCSD(T), and CCSDT PES, respectively. In all cases, it is clearly evident an increasing blueshift and full width at half maximum (FWHM) of the absorption peak upon in- 
crease of the number of $\mathrm{He}$ atoms in the clusters. This finding is in line with what was previously found for helium clusters doped with alkali metals ${ }^{8}$ and with atomic silver. ${ }^{7}$ However, different from the $\mathrm{AgHe}_{n}$ case, no redshift is present for the small clusters. This result, in conjunction with the relative shape of the ground and excited $\mathrm{MgHe}$ potentials, suggests that the direct formation of exciplexes ${ }^{13}$ during the vertical excitation process is quite unlikely. We also notice that the magnitude of the blueshift is larger for $\mathrm{Mg}$ than in the case of alkali-doped helium clusters, ${ }^{8}$ indicating that the excited $\mathrm{Mg}$ atom finds itself in a more repulsive environment, and the characteristic long tail of floating dopant is absent even in the $\operatorname{CCSD}(\mathrm{T})$ results.

Despite the similar trend observed upon increasing the number of He atoms, the three sets of spectra present several interesting differences for a given cluster size; both the blueshift and the FWHM increase in the sequence $\operatorname{CCSD}(\mathrm{T})$ $<$ CCSDT $<$ MP4. Furthermore, the CCSD $(T)$ and CCSDT sets of results show a spectral shape different from the absorption curve obtained with the MP4 for some of the medium-size droplets, the MP4 spectra presenting a shoulder in the absorption curve at large wave numbers already for $\mathrm{MgHe}_{30}$. This feature is evident in the CCSDT case only starting from $\mathrm{MgHe}_{40}$, and is never present in the $\operatorname{CCSD}(\mathrm{T})$ results.

Given the previous discussion on the dopant location obtained from the simulations, it is straightforward to suggest that this shoulder is due to the onset of a more compact and complete first solvation shell of Mg at $n=30$ for MP4 and at $n=40$ for CCSDT. A similar explanation was previously proposed to rationalize the increase in blueshifts and in FWHM in $\mathrm{AgHe}_{n}{ }^{7}$ More precisely, we recall that some of the DMC simulations generated an anisotropic He environment around the dopant. In these systems, Mg resides preferentially in a deep dimple on the cluster surface, and the local environment allows the excited ${ }^{1} P$ state to orient itself so that the $3 p$ orbital containing the excited electron is directed outside the cluster. As a consequence of this freedom, the repulsive interaction between the ${ }^{1} P \mathrm{Mg}$ state and the local He environment is decreased (we recall that the $\Sigma$ excited state is highly repulsive), and so is the shift of the component of the spectral line due to the $\Sigma$ state. ${ }^{46}$ In turn, this delays the onset of the shoulder at large wave numbers until the complete solvation has occurred.

The location of the maximum in the spectra can be used to better quantify the physical picture of the vertically excited $\mathrm{Mg}$ and to compute its binding energy to the cluster using the formula $E_{\text {bind }}^{\mathrm{exc}}=E_{\mathrm{bind}}^{\mathrm{gs}}-\left(h \nu_{\text {cluster }}-h \nu_{\mathrm{vac}}\right)$. Extracting the position of the maxima for the simulations on $\mathrm{MgHe}_{50}$ and using the data in Table III, one obtains $E_{\text {bind }}^{\text {exc }}($ MP4 $)$ $\simeq-518 \mathrm{~cm}^{-1}, \quad E_{\text {bind }}^{\text {exc }}($ CCSDT $) \simeq-589 \mathrm{~cm}^{-1}, \quad$ and $E_{\text {bind }}^{\text {exc }} \operatorname{CCSD}(\mathrm{T}) \simeq-235 \mathrm{~cm}^{-1}$, clearly indicating the instability of the excited $\mathrm{Mg}$ atom attached to the clusters. In turn, these results suggest that the breakup of the excited system into the excited $\mathrm{Mg}$ atom and the ground-state He cluster is a likely dissociation channel, and that the excited $\mathrm{Mg}$ could leave the droplet converting some of the excess electronic energy into kinetic energy. On the other hand, another possible outcome following the vertical excitation is represented by a rear- rangement of the He atoms surrounding the excited dopant; these atoms would adjust their distribution to reflect the new shape of the interaction potential and to produce a more anisotropic, but also more strongly bound, environment around the Mg. During this process, some of the excess energy contained in the system after the excitation must be somehow dissipated, and it is likely that this would produce a partial breakup of the He moiety. Unfortunately, it is difficult to predict the likelihood of this process using exclusively our DMC results, and a dynamical simulation of the relaxation process of the He cluster appears mandatory to clarify this detail.

Comparing the experimental spectra reported in Refs. 13 and 14 with the simulation results obtained for our largest clusters [collected together in Fig. 9(c)], several interesting observations can be made. First, all the results show a good agreement concerning the location of the two experimental spectra, once again indicating the good accuracy of the DIM model to approximate the interaction energy for the dopant excited state. However, the shape of the MP4 and CCSDT spectra for $\mathrm{MgHe}_{50}$ is much closer to the experimental bulk-He spectrum ${ }^{13}$ than to the one obtained in nanodroplets $;{ }^{14}$ the latter presents a convex shape in the region of 35 800-36500 $\mathrm{cm}^{-1}$ where the bulk spectrum shows a concave behavior (henceforth, a "shoulder"). It is also interesting to note that none of our simulation results present an absorption shape similar to the cluster spectra in the region of 35 300-35800 cm $\mathrm{cm}^{-1}$, where the experimental results were fitted using two Gaussians; this feature was explained invoking the quadrupolar deformation of the $\mathrm{Mg}$ cavity similar to what was previously found for heavy alkali and Ag. Since it was previously shown that coupling the DIM method with DMC or PIMC simulations ${ }^{7}$ accurately describes the features due to the anisotropy in the local dopant environment, at this stage it is difficult for us to propose an explanation for the lack of structure found in the range of $35300-35800 \mathrm{~cm}^{-1}$. The possibility to invoke the presence of a vibronic coupling between the $\mathrm{He}$ atoms and the $\mathrm{Mg}$ electrons during the excitation process seems to be ruled out by the lack of similar features in the bulk-He spectrum. As an alternative, we can conceive that the different shape of the excitation spectra in nanodroplets and bulk may be due to the two different detection mechanisms used; this implies that the shape of the fluorescent emission is strongly dependent on the wavelength used to excite the chromophore, though.

On the computational side, we cannot rule out a priori the possibility that the absence of structure in the excitation spectrum may be due to size effects and that larger clusters may indeed have this very feature. However, we note that in the case of the MP4 potential-where the cluster structure converges quite rapidly with the number of $\mathrm{He}$ atoms - the spectral shape obtained for $\mathrm{MgHe}_{30}, \mathrm{MgHe}_{40}$, and $\mathrm{MgHe}_{50}$ is very similar to each other, and presents a single absorption peak. Cluster size effects might occur using the $\operatorname{CCSD}(\mathrm{T})$ and CCSDT potentials, where the structural parameters of $\mathrm{MgHe}_{n}$ are more critically dependent on the number of $\mathrm{He}$ atoms. 


\section{Analysis of the solvation parameters}

The DMC simulation results presented in the previous sections have highlighted a dependency of the solubility and the spectral properties of $\mathrm{Mg}$ in $\mathrm{He}_{n}$ clusters on the shape of the interaction curve for $6 \leqslant n \leqslant 50$. However, a deeper quantitative understanding of the underlying causes producing such distinct behaviors is still missing, in spite of the accurate numerical results. In particular, we would like to understand which specific feature, if any, in the three potentials is primarily responsible for the change in solubility highlighted by the $\mathrm{Mg}$-gc distance and $\cos (\mathrm{HeMgHe})$ distribution functions.

To shed some light on the peculiarities of this phenomenon, we turned to an exact formulation of DFT as a way to extract energetic quantities that are expected to play an important role in defining the impurity solvation. To this end, we extended the exact DFT formalism for homogeneous systems proposed by Levy et $a l^{47}$ to describe an impurity attached to a homogeneous cluster. More specifically, we derived an effective one-particle Schrödinger equation for the $\mathrm{Mg}$-gc radial probability density and the $\mathrm{Mg}$ binding energy $\mu$ to the droplet containing the effective potential

$$
V_{\text {eff }}\left(\mathbf{R}_{\mathrm{gcMg}}\right) \simeq V_{\mathrm{eff}}^{\mathrm{HeHe}}\left(\mathbf{R}_{\mathrm{gcMg}}\right)+V_{\mathrm{eff}}^{\mathrm{MgHe}}\left(\mathbf{R}_{\mathrm{gcMg}}\right),
$$

where

$$
V_{\text {eff }}^{\mathrm{HeHe}}\left(\mathbf{R}_{\mathrm{gcMg}}\right)=\frac{\left\langle\Phi\left|V_{\mathrm{HeHe}}\left(\mathbf{y}_{\mathrm{He}}\right)\right| \Phi\right\rangle_{n-1}}{\langle\Phi \mid \Phi\rangle_{n-1}}
$$

and

$$
V_{\mathrm{eff}}^{\mathrm{MgHe}}\left(\mathbf{R}_{\mathrm{gcMg}}\right)=\frac{\left\langle\Phi\left|V_{\mathrm{MgHe}}\left(\mathbf{y}_{\mathrm{He}}, \mathbf{R}_{\mathrm{gcMg}}\right)\right| \Phi\right\rangle_{n-1}}{\langle\Phi \mid \Phi\rangle_{n-1}} .
$$

Here, $\mathbf{y}_{\mathrm{He}}$ indicates the $3(n-1)$ dimensional vector describing the $\mathrm{He}$ atoms position with respect to the center of the $\mathrm{He}$ moiety and $\langle\cdots\rangle_{n-1}$ represents the average over $\mathbf{y}_{\mathrm{He}}$. The complete derivation of this equation is available via the EPAPS deposit service. ${ }^{48}$ Here, we only mention that the two terms in the right-hand side of Eq. (9) represent the average interaction of the He atoms among themselves and with $\mathrm{Mg}$, as a function of the position of the dopant in the cluster. Both quantities can be exactly or accurately computed with DMC simulations.

Figure 10 shows the radial dependency of the angular average of $V_{\text {eff }}\left(\mathbf{R}_{\mathrm{gcMg}}\right), V_{\mathrm{eff}}^{\mathrm{HeHe}}\left(\mathbf{R}_{\mathrm{gcMg}}\right)$, and $V_{\text {eff }}^{\mathrm{MgHe}}\left(\mathbf{R}_{\mathrm{gcMg}}\right)$ obtained during the simulation of $\mathrm{MgHe}_{30}$ with the MP4 and $\operatorname{CCSD}(\mathrm{T})$ pair potentials. Basing on the results shown in Fig. 7 , this cluster and these two PESs were chosen as representative cases of the possible different behaviors.

The computed $V_{\mathrm{eff}}^{\mathrm{MgHe}}$ values decrease for $\left\|\mathbf{R}_{\mathrm{gcMg}}\right\| \rightarrow 0$, and this is due to the increased number of He atoms surrounding the dopant; ${ }^{3}$ in turn, this indicates the increased stabilization of the impurity when brought inside the cluster. $V_{\mathrm{eff}}^{\mathrm{MgHe}}$ also shows a parabolic shape when $\left\|\mathbf{R}_{\mathrm{gcMg}}\right\| \simeq 0$, strongly suggesting that the analysis carried out by Lehmann $^{3}$ is valid for small clusters. Two differences are, however, present for the two PESs, namely a global shift in value reflecting the difference in the well depth between

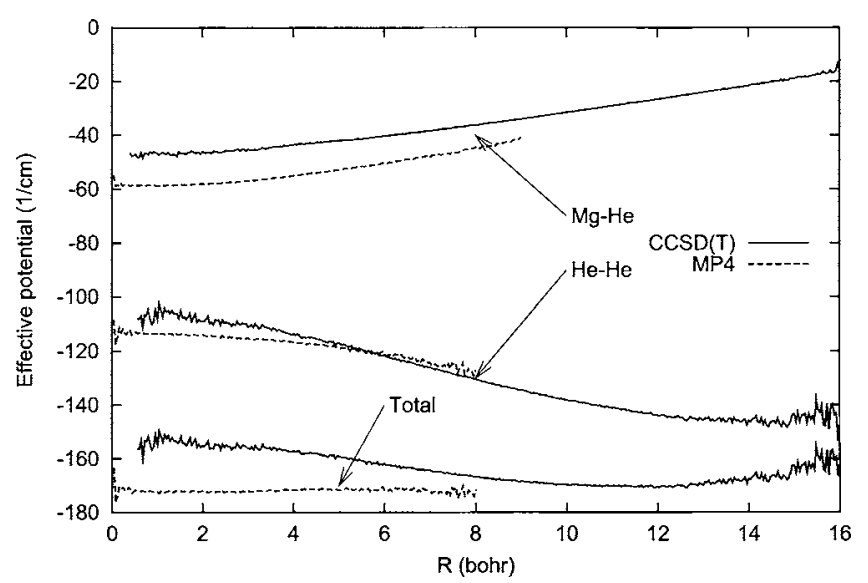

FIG. 10. Radial dependency of the angular average of the total effective potential $V_{\text {eff }}$ and of its components $V_{\text {eff }}^{\mathrm{HeHe}}$ and $V_{\mathrm{eff}}^{\mathrm{MgHe}}$ for $\mathrm{MgHe}_{30}$ using the MP4 and $\operatorname{CCSD}(\mathrm{T})$ potential curves.

MP4 and $\operatorname{CCSD}(\mathrm{T})$, and a larger curvature for the MP4 results; the latter indicates a tighter binding of the impurity to the cluster center in the MP4 case.

At variance with $V_{\mathrm{eff}}^{\mathrm{MgHe}}, V_{\mathrm{eff}}^{\mathrm{HeHe}}$ increases in value when the impurity is closer to the geometrical center of the $\mathrm{He}$ moiety, a finding that can be easily explained by recalling that the He atoms lose stabilizing interactions due to the formation of a cavity inside the cluster and to the increase of the cluster surface area. Similar to the discussion given by Lehmann ${ }^{4}$ on the so-called "buoyancy correction," we also found that the $\mathrm{He}$ atoms lose more stabilizing interactions when the impurity sits close to the cluster center than when it is just below the surface of the droplet. In this respect, the $\operatorname{CCSD}(\mathrm{T})$ results show a steeper increase in $V_{\mathrm{eff}}^{\mathrm{HeHe}}$ than the MP4 ones, the most likely explanation for this feature being the different effective radius for $\mathrm{Mg}$ produced by the two potentials. Defining this radius as the distance at which a given PES has a value of zero, we found that the $\operatorname{CCSD}(\mathrm{T})$ PES is larger by roughly 0.2 bohr than the MP4 one.

Due to the different features of $V_{\mathrm{eff}}^{\mathrm{HeHe}}$ and $V_{\mathrm{eff}}^{\mathrm{MgHe}}$ as a function of the interaction curve, $V_{\text {eff }}$ shows two qualitatively different shapes: the $\operatorname{CCSD}(\mathrm{T})$ potential presents an overall repulsive interaction from the cluster surface inward due to the large values of $V_{\mathrm{eff}}^{\mathrm{HeHe}}$, while the MP4 potential shows an almost complete cancellation between the two components and produces a shallow attractive interaction between the dopant and the center of the He moiety. In the latter case, it should be stressed that both the larger interaction between $\mathrm{Mg}$ and $\mathrm{He}$ and the smaller size of $\mathrm{Mg}$ play a relevant role in defining the shape of the total effective potential. $V_{\text {eff }}$ was also computed using the MP4 curve for $\mathrm{MgHe}_{12}, \mathrm{MgHe}_{20}$, $\mathrm{MgHe}_{30}$, and $\mathrm{MgHe}_{40}$ and gave a qualitatively correct prediction of the solvation phenomenon. Indeed, whereas it presents a deep minimum away from the droplet center in the small clusters, a shallow minimum close the center of the He moiety is produced upon increasing the system size.

\section{CONCLUSIONS}

In this work we presented a computational study of the ground-state properties and excitation spectrum of $\mathrm{Mg}$ at- 
tached to $\mathrm{He}_{n}$ clusters $(n=2-50)$. This study was carried out using the exact DMC method in conjunction with accurate model potentials written as a sum over pair interactions, a commonly used and fairly accurate approximation for weakly interacting systems. For the $\mathrm{He}-\mathrm{He}$ interaction potential we used the well known and widely employed TTY model, ${ }^{19}$ whereas large basis set ab initio MP4 and $\operatorname{CCSD}(\mathrm{T})$ energies were computed and interpolated using quintic splines to produce two accurate potentials for the $\mathrm{Mg}-\mathrm{He}$ pair. Additionally, a third $\mathrm{Mg}-\mathrm{He}$ model potential was obtained by interpolating the CCSDT interaction energies computed by Hinde. ${ }^{17}$ The well depth and equilibrium distance of the ground-state PES are $-5.70 \mathrm{~cm}^{-1}$ and $5.03 \AA$ for MP4, $-4.74 \mathrm{~cm}^{-1}$ and $5.11 \AA$ for $\operatorname{CCSD}(\mathrm{T})$, and $-5.01 \mathrm{~cm}^{-1}$ and $5.07 \AA$ for CCSDT computations.

We computed total evaporation and $\mathrm{Mg}$ binding energies as a function of $n$ using the three interaction curves; the comparison between the results for $\mathrm{MgHe}_{40}$ and $\mathrm{MgHe}_{50}$ indicates an almost converged $\mathrm{He}$ environment around the atomic dopant for $n=50$ in the case of the MP4 and CCSDT potentials. In spite of the similar shape of the interaction curves (Fig. 1), the results of the DMC simulations revealed markedly different solubilities of $\mathrm{Mg}$ in $\mathrm{He}_{n}$ as a function of both the $\mathrm{Mg}-\mathrm{He}$ pair interaction and $n$ : the MP4 PES suggests that $\mathrm{Mg}$ is located at the center of $\mathrm{He}_{n}$ independent of $n ; \operatorname{CCSD}(\mathrm{T})$ always predicts a surface location for the dopant; and CCSDT, instead, presents a change in Mg behavior around $n=25$, predicting $\mathrm{Mg}$ to be soluble in the larger clusters. These results highlight both a marked sensitivity of the solvation properties with respect to the global shape of the PES and the ability of DMC to discriminate qualitatively different behaviors generated by subtle differences in the interaction potentials. To gain a deeper understanding of the solvation process in these species, we used a modified version of the DFT approach proposed in Ref. 47 and found that both the well depth and the position of the repulsive wall influence the solubility of $\mathrm{Mg}$ in $\mathrm{He}_{n}$.

Our simulation results bear relevance also with respect to the solvation model proposed by Ancilotto et al. ${ }^{5}$ This model predicts solvation whenever the dimensionless parameter $\lambda$ exceeds 1.9, whereas our results present a quite different scenario; the simulations using the CCSD(T) PES strongly suggest that solvation is not automatically assured in spite of the large $\lambda\left[\lambda_{\mathrm{CCSD}(\mathrm{T})}=2.66\right]$. Furthermore, the dopant solubility may depend on the size of the cluster (as highlighted by the CCSDT simulations) and may be sensitive to subtle differences between interaction potentials [for instance, compare the CCSD(T) and CCSDT cases]. In turn, these findings stress that highly accurate pair interactions are needed to correctly predict the solubility of neutral dopants with isotropic pair potential.

We found a dependency on the shape of the PES also for the simulated excitation spectra, the solvated Mg (MP4 and CCSDT cases) showing a broader and more blueshifted absorption band than the floating dopant $[\operatorname{CCSD}(\mathrm{T})]$. Different from the results obtained for $\mathrm{AgHe}_{n}{ }^{7}$, no redshifted bands were found for the small $\mathrm{MgHe}_{n}$ clusters, suggesting that the formation of exciplexes would require a reorganization of the He environment around $\mathrm{Mg}$ following the vertical excitation.
Indeed, this outcome is considered quite likely by Reho et $a l .{ }^{14}$ on the basis of their time-resolved results, and it would be interesting to study the dynamical changes involved during this process.

Comparing the simulated and experimental cluster spectra we found a good agreement between the position of the excitation bands, once more validating the usage of the DIM method to approximate the excited PES. In spite of this agreement, there is a marked difference in shape between the experiments and the results obtained by theory for $\mathrm{MgHe}_{50}$; whereas the simulated spectra always present a smooth band without peculiar features, the experimental spectrum shows a two-peak structure interpreted as the outcome of a quadrupolar deformation of the He cage around the solvated $\mathrm{Mg}$. Bearing in mind that DMC was previously found to describe correctly this kind of deformations, ${ }^{7}$ this discrepancy calls for further theoretical and experimental investigations.

\section{ACKNOWLEDGMENTS}

One of the authors (M.M.) acknowledges Dario Bressanini for independently testing some of the simulation results and for providing a more precise DMC energy for $\mathrm{He}_{40}$, and the EPSRC-UK for an Advanced Research Fellowship (GR/ R77803/01).

${ }^{1}$ J. P. Toennies and A. F. Vilesov, Angew. Chem., Int. Ed. 43, 2622 (2004).

${ }^{2}$ E. Lee, D. Farrelly, and K. B. Whaley, Phys. Rev. Lett. 83, 3812 (1999).

${ }^{3}$ K. K. Lehmann, Mol. Phys. 97, 645 (1999).

${ }^{4}$ K. K. Lehmann, Mol. Phys. 98, 1991 (2000).

${ }^{5}$ F. Ancillotto, P. B. Lerner, and M. W. Cole, J. Low Temp. Phys. 101, 1123 (1995).

${ }^{6}$ S. Ogata, J. Phys. Soc. Jpn. 68, 2153 (1999).

${ }^{7}$ M. Mella, M. C. Colombo, and G. Morosi, J. Chem. Phys. 117, 9695 (2002).

${ }^{8}$ A. Nakayama and K. Yamashita, J. Chem. Phys. 114, 780 (2001).

${ }^{9}$ M. Casalegno, M. Mella, G. Morosi, and D. Bressanini, J. Chem. Phys. 112, 69 (2000).

${ }^{10}$ D. Bressanini, M. Zavaglia, M. Mella, and G. Morosi, J. Chem. Phys. 112, 717 (2000).

${ }^{11}$ F. Sebastianelli, C. Di Paola, I. Baccarelli, and F. A. Gianturco, J. Chem. Phys. 119, 8276 (2003).

${ }^{12}$ F. Paesani, F. A. Gianturco, and K. B. Whaley, J. Chem. Phys. 115, 10225 (2001).

${ }^{13}$ Y. Moriwaki and N. Morita, Eur. Phys. J. D 5, 53 (1999).

${ }^{14}$ J. Reho, U. Merker, M. R. Radcliff, K. K. Lehmann, and G. Scoles, J. Chem. Phys. 112, 8409 (2000).

${ }^{15}$ F. Stienkemeier, F. Meier, and H. O. Lutz, J. Chem. Phys. 107, 10816 (1997).

${ }^{16}$ H. Partridge, J. R. Stallcop, and E. Levin, J. Chem. Phys. 115, 6471 (2001).

${ }^{17}$ R. J. Hinde, J. Phys. B 36, 3119 (2003).

${ }^{18}$ F. O. Ellison, J. Am. Chem. Soc. 85, 3540 (1963).

${ }^{19}$ K. Tang, J. P. Toennies, and C. L. Yiu, Phys. Rev. Lett. 74, 1546 (1995).

${ }^{20}$ E. Czuchaj, H. Stoll, and H. Preuss, J. Phys. B-At. Mol. Opt. 20, 1487 (1987).

${ }^{21}$ D. J. Funk, W. H. Breckenridge, J. Simon, and G. Chalasinski, J. Chem. Phys. 91, 1114 (1989).

${ }^{22}$ Q. Hui, Ph.D. thesis, Graduate School of Science and Engineering, Saitama University, 1997.

${ }^{23}$ U. Kleinekathöfer, Chem. Phys. Lett. 324, 403 (2000).

${ }^{24}$ S. Huzinaga, M. Klobakowski, and H. Tatewaki, Can. J. Chem. 63, 1812 (1985).

${ }^{25}$ D. Woon and T. Dunning, J. Chem. Phys. 100, 2975 (1994).

${ }^{26}$ F.-M. Tao and Y.-K. Pan, J. Chem. Phys. 97, 4989 (1992).

${ }^{27}$ M. W. Schmidt, K. K. Baldridge, J. A. Boatz et al., J. Comput. Chem. 
14, 1347 (1993); A. A. Granovsky, http://lcc.chem.msu.su/gran/ gamess-index.old.html

${ }^{28}$ M. J. Frisch, G. W. Trucks, H. B. Schlegel et al., Gaussian98, Revision A.11.1, Gaussian, Inc., Pittsburgh, PA, 2001.

${ }^{29}$ S. F. Boys and F. Bernardi, Mol. Phys. 19, 553 (1970).

${ }^{30}$ A. D. McLean and G. S. Chandler, J. Chem. Phys. 72, 5639 (1980).

${ }^{31}$ P. O. Widmark, B. J. Persson, and B. Roos, Theor. Chim. Acta 79, 419 (1991).

${ }^{32}$ E. F. Archibong and A. J. Thakkar, Phys. Rev. A 44, 5478 (1991).

${ }^{33}$ S. M. Cybulski and R. R. Toczylowski, J. Chem. Phys. 111, 10520 (1999).

${ }^{34}$ W. C. Martin, J. Phys. Chem. Ref. Data 9, 1 (1980).

${ }^{35}$ B. L. Hammond, J. W. A. Lester, and P. J. Reynolds, Monte Carlo Methods in Ab Initio Quantum Chemistry (World Scientific, Singapore, 1994).

${ }^{36}$ S. W. Rick, D. L. Lynch, and J. D. Doll, J. Chem. Phys. 95, 3506 (1991).

${ }^{37}$ D. Bressanini, G. Morosi, and M. Mella, J. Chem. Phys. 116, 5345 (2002).

${ }^{38}$ E. Cheng and K. B. Whaley, J. Chem. Phys. 104, 3155 (1996).

${ }^{39}$ M. Lax, J. Chem. Phys. 20, 1752 (1952).

${ }^{40}$ F. Paesani, F. A. Gianturco, and K. B. Whaley, J. Chem. Phys. 115, 10225 (2001).

${ }^{41}$ D. Blume, M. Lewerenz, F. Huisken, and M. Kaloudis, J. Chem. Phys.
105, 8666 (1996)

${ }^{42}$ E. Sola, J. Casulleras, and J. Boronat, J. Low Temp. Phys. 134, 787 (2004).

${ }^{43}$ S. Cozzini and M. Ronchetti, Phys. Rev. B 53, 12040 (1996).

${ }^{44}$ F. Sebastianelli, I. Baccarelli, C. Di Paola, and F. A. Gianturco, J. Chem. Phys. 119, 5570 (2003).

${ }^{45}$ Assuming the liquid He droplet to behave as an uncompressible fluid, it can be shown that the increase in the droplet surface $S$ due to the solvation of a rigid impurity or radius $r$ is given by $\Delta S(V, r)$ $=4 \pi V^{2 / 3}\left\{\left[3 /(4 \pi)+r^{3} / V\right]^{2 / 3}-[3 /(4 \pi)]^{2 / 3}\right\}$, where $V$ is the original volume of the droplet. For small $r^{3} / V$ ratios, $\Delta S(V, r)$ can be shown to decay as $V^{-1 / 3}$ indicating a smaller surface increase for larger $V$.

${ }^{46}$ We tested this idea by decomposing the spectrum in its three components due to the three eigenvalues obtained from the DIM matrix. Invariably, we found that the one with the largest blueshift was primarily due to the $\Sigma$ state interactions and that it was responsible for the right shoulder on the spectrum peak.

${ }^{47}$ M. Levy, J. P. Perdew, and V. Sahni, Phys. Rev. A 30, 2745 (1984).

${ }^{48}$ See EPAPS Document No. E-JCPSA6-123-021526 for the document presenting the complete derivation of Eq. (9). This document can be reached through a direct link in the online article's HTML reference section or via the EPAPS homepage (http://www.aip.org/pubservs/epaps.html). 